\title{
1 Porous medium modeling and parameter sensitivity analysis of \\ 2 1-MCP distribution in boxes with apple fruit
}

3 Alemayehu Ambaw ${ }^{(a)}$, Pieter Verboven ${ }^{(a)}$, Thijs Defraeye ${ }^{(a)}$, Engelbert Tijskens ${ }^{(a)}$, Ann Schenk

$4 \quad{ }^{(a)}$, Umezuruike Linus Opara ${ }^{(b)}$, Bart M. Nicolai ${ }^{\left(a, c^{*}\right)}$

5 a BIOSYST-MeBioS, Katholieke Universiteit Leuven, Willem de Croylaan 42, B-3001 Leuven,

6 Belgium

$7 \quad{ }^{b}$ Postharvest Technology Research Laboratory, South African Chair in Postharvest Technology,

8 Faculty of AgriSciences, Stellenbosch University, Private Bag X1, Stellenbosch 7602, South

9 Africa

$10{ }^{\mathrm{c}}$ Flanders Centre of Postharvest Technology, Willem de Croylaan 42, B-3001 Leuven, Belgium

$11 *$ *orresponding author; email: bart.nicolai@ biw.kuleuven.be.

\section{Abstract}

13 1-methylcyclopropene (1-MCP) is a gas used commercially in cold storage rooms to delay

14 ripening of fruit. It is currently not known how the gas distributes in the bins and is taken up by

15 the fruit. Here a porous-medium model is developed based on volume averaging of the transport

16 equations of momentum and 1-MCP in the air and product. The proposed model was compared

17 with experimental data and validated direct-CFD model. The velocity field in and around the

18 stack were well reproduced by the porous medium model. Concentration predictions were in

19 average $4 \%$ and maximally $7 \%$ over the average measured values. The porous medium model

20 also agrees well with the direct-CFD model. Influential model parameters were identified through

21 parameter sensitivity study. Porosity, skin mass transfer coefficient and specific surface area 
22 strongly affect the simulation. Accordingly model simplifications were suggested and

23 recommended for commercial cold storage rooms.

\section{Keywords:}

25 Postharvest, Fruit ripening, adsorption kinetics, porous medium, CFD, cold storages

\section{Introduction}

27 1-MCP is applied in gas form as a fumigant by placing a proprietary 1-MCP release system in a

28 room filled with produce. Through a combination of diffusion and convection the 1-MCP gas

29 reaches the fruit and is bound on specific binding sites to delay ripening by blocking the action of

30 ethylene (Blankenship \& Dole, 2003; Sisler \& Blankenship, 1996). 1-MCP is used at low

31 concentrations $\left(2.5 \mathrm{~nL} \mathrm{~L}^{-1}\right.$ to $\left.1 \mu \mathrm{L} \mathrm{L}^{-1}\right)$. The efficacy varies with the commodity, duration of

32 treatment, temperature and method of application (Blankenship \& Dole, 2003). To date, there is

33 limited understanding of effective dose and distribution of 1-MCP to maximize profits in

34 agribusiness to provide quality consumer products (Blankenship \& Dole, 2003).

35 There are relatively few studies on the distribution of 1-MCP gas in loaded cool storage rooms.

36 While the gas has a high diffusion coefficient (Ambaw et al. 2011), there are reasons that could

37 cause an ineffective distribution of the gas in the cool room. First, wooden and cardboard bin and

38 bin liner materials have a significant capacity to bound 1-MCP and can potentially reduce the

39 active substance concentration in the air (Vallejo and Beaudry, 2006; Ambaw et al., 2011).

40 Second, air circulation controls the convection driven distribution of the gas and could provide

41 preferential routes while particular zones are not reached. Third, air flow may add to the rate at

42 which the gas is transferred to the fruit and thus the rate at which the fruit is saturated by $1-\mathrm{MCP}$

43 (Ambaw et al. 2012a). Thus, the gas transport process with the dimensions and configuration of 
44 the cool storage room can play an important role in the efficacy of the treatment. To study the

45 effect of these interacting factors experimentally is difficult and time consuming. Numerical 46 models, therefore, are a valuable tool to understand and design the process. In postharvest and

47 various other processes, computational fluid dynamics (CFD) is commonly applied as an

48 alternative to experimental studies of convection driven problems (Ambaw et al. 2012a,b; Delele

49 et al. 2010; Alvarez and Flick, 2007; Verboven et al. 2006; Zou et al., 2006a,b).

50 Inside the fruit, 1-MCP diffuses from the surface to the centre and is absorbed by target and non-

51 target binding sites. A model that describes this diffusion-reaction process in fruit tissue and non-

52 target solid materials was developed by Ambaw et al. (2011). This model was employed in a

53 direct CFD model of 1-MCP applications in which the detailed geometry of apple fruit was

54 considered (Ambaw et al., 2012a). Simulations with this direct CFD model aided the detailed

55 analysis of the effect of design and operational factors when the number of fruit was small

56 enough to be handled numerically by desk-top computers. Direct CFD modeling, resolving every

57 fruit, is impractical for fully loaded cool storage rooms filled with hundreds of tons of fruit

58 (Verboven et al., 2006). Here, dimensions range from one-tenths of a millimeter to several

59 meters. The wide range of scales requires a large number of computational points in the

60 numerical procedure to solve the direct CFD model, increasing the computational demand

61 beyond the resources available today. A porous medium model using volume-averaged transport

62 equations is hence required (Hoang et al., 2003, 2004; Nahor et al., 2005).

63 In the volume averaging technique, the products and the air spaces in between compose a porous

64 domain. Every volume within the domain is assumed to contain the product (the solid phase) and

65 the air (the fluid phase). The two phases are continuum, separated by irregular interfaces that are 66 not resolved geometrically but parameterized through the volume averaging procedure 
67 (Hassanizadeh and Gray, 1979). Depending on the level of simplification, one can arrive at one68 phase or two-phase models (Verboven et al., 2006). The one-phase model assumes local 69 thermodynamic equilibrium and arrives at a single transport equation, but the simplifications 70 applied in using this approach are so severe that it usually leads to considerable errors, especially 71 for transient problems (Lerew, 1978). The two-phase model involves volume-averaging of the 72 transport equations over each phase to arrive at system of equations to be solved separately. The 73 equations are coupled through interfacial conditions which are expressed as function of 74 interfacial concentrations and interfacial fluxes. This approach is more realistic for transient 75 problems where the local equilibrium hypothesis is not well verified (Verboven et al. 2006). The 76 two-phase volume-averaging has been implemented for postharvest applications to solve heat and 77 mass transfer problems, including room design and package design for optimal cooling of

78 horticultural products (Hoang et al., 2003, 2004, Nahor et al., 2005). However, existing porous 79 medium models of airflow and mass transfer in stacked fruits have not incorporated diffusion80 adsorption phenomena.

81 In the present paper, the two-phase volume averaging technique is implemented to develop a 82 porous medium model of the spatial and temporal 1-MCP distribution in cool storage rooms that 83 take into account the gas diffusion-adsorption phenomena. A previously developed and validated 84 direct CFD model (Ambaw et al. 2012a) allowed carrying out a detailed evaluation of the 85 approach. A parameter sensitivity analysis is performed to study the relative importance of the 86 model parameters. 


\section{$87 \quad 2$ Materials and methods}

\section{$88 \quad 2.1$ Fruit supply}

89 'Jonagold' apples (Malus domestica Borkh, cv. Jonagold) were purchased directly after harvest

90 from a local grower in Belgium in September, 2011. All the fruits used for the test were free of

91 visual defects. Fruits were stored at $1^{\circ} \mathrm{C}$ in normal atmospheric air before and during the

92 experiment that took place within a few days. The mean \pm standard deviation $(n=10)$ of the

93 mass and volume of fruits were $235 \mathrm{~g} \pm 32 \mathrm{~g}$ and $281 \mathrm{~mL} \pm 36 \mathrm{~mL}$, respectively.

\section{$94 \quad 2.2$ Experiment in the $500 \mathrm{~L}$ container}

95 The experiment was designed to obtain spatio-temporal 1-MCP concentration data and spatial 96 velocity data. For this, $80 \mathrm{~kg}$ fruit (in four plastic boxes, each holding 20kg) and a 1-MCP

97 releasing system were placed together in a closed 500L container (see Fig.1). At regular intervals 98 during a 24 hours period, gas samples were taken from eight locations and 1-MCP concentration 99 measurement was made for each sample using gas chromatography. The times of 1-MCP 100 concentration measurements were 2, 4, 6, 8, 10 and $24 \mathrm{~h}$ after closing the container. Velocity 101 measurements were done by means of air velocity transducers from a total of twenty-five 102 different locations inside the container. The experiment was performed in three replicates. Details 103 of the experimental setup were discussed in our previous work (Ambaw et al., 2012a) and hence 104 not repeated here.

\section{$105 \quad 2.3$ Mathematical model}

106 A two-phase porous medium model of the convection-diffusion-reaction of 1-MCP in stacks of 107 fruit was developed using the volume averaging technique as described by several researchers 108 (Hassanizadeh and Gray, 1979; Liu and Masliyah, 2005, Quintard and Whitaker, 2005; Whitaker, 
109 1999) and are hence not repeated here. The general and particular assumptions taken to apply the

110 volume averaging technique and the resulting model equations are stated below.

\section{$111 \quad$ 2.3.1 Assumptions}

112 - Fruit loaded boxes were assumed as a porous domain consisting of a fluid phase (1-MCP-

113 air mixture) and a solid phase (apple fruit).

114 - Passive scalar transport equations were used to describe the transport of 1-MCP in air.

115 This is reasonable for the fact that 1-MCP used during treatment is extremely dilute; its

116 effect on the airflow was, therefore, negligible.

117 1-MCP adsorption was assumed irreversible and the bound 1-MCP is assumed to be a 118 non-convective, non-diffusive scalar variable. Unbound 1-MCP exists in the air outside 119 the solid as well as in the intercellular pore spaces of the fruit. The transport of unbound

120 1-MCP in air was described by a convective-diffusive scalar transport equation and by a 121 diffusive scalar transport equation in the solid pores. Convection-diffusion-adsorption 122 parameters obtained from previously developed and validated models (Ambaw et al. 123 2011) were used.

124 - Isothermal conditions were assumed. Fruit was stored at $1{ }^{\circ} \mathrm{C}$ in normal atmospheric air 125 before and during the experiment. At this low temperature, the respiration heat generation 126 of fruit is considerably low and was neglected.

127 - The product is assumed homogeneous with constant properties throughout. 
- The product is assumed spherical and the specific area of the packed apple fruit was estimated based on porosity and average diameter of the spheres representing the apple fruit.

- The pressure loss inside the porous medium was described by directional loss models with linear and quadratic coefficients determined from simulated pressure drop data from direct CFD model of single fruit loaded box (Delele et al., 2009).

\section{$134 \quad$ 2.3.2 Macroscopic equation of airflow}

135 The porosity, mass conservation and momentum conservations for the porous domain are given

136 by Eqn. 1, 2 and 3, respectively (ANSYS CFX-Solver Theory Guide), assuming constant porosity

137 in space:

$138 d V_{a}=\varepsilon d V$

$139 \nabla \cdot(\varepsilon \mathbf{U})=0$

$140 \frac{\partial\left(\varepsilon \rho_{a} \mathbf{U}\right)}{\partial t}+\nabla \cdot\left(\varepsilon \rho_{a}(\mathbf{U} \otimes \mathbf{U})\right)-\nabla \cdot\left(\varepsilon\left(\mu+\mu_{t}\right)\left(\nabla \mathbf{U}+(\nabla \mathbf{U})^{\boldsymbol{\top}}\right)-\frac{2}{3} \delta \nabla \cdot \mathbf{U}\right)=\varepsilon S_{M}-\varepsilon \nabla p$

$141 S_{M}$ in Eqn. (3) calculates the pressure loss of the flow in the porous medium. In this study the 142 pressure loss was modeled using directional loss model as:

$143 \quad S_{M, i}=C_{R 1 i} u_{i}+C_{R 2 i}|\boldsymbol{U}| u_{i}$

\section{$144 \quad$ 2.3.3 Macroscopic equation of 1-MCP conservation}

145 The fluid and solid phase volume-averaged unbound 1-MCP transport equation are given by:

$146 \frac{\partial\left(\varepsilon C_{a}\right)}{\partial t}+\nabla \cdot\left(\varepsilon C_{a} \mathbf{U}-\tau \varepsilon\left(D_{a}+D_{t}\right) \nabla C_{a}\right)+a_{s} h_{m}\left(C_{a}-C_{s}\right)=0(5)$ 
$147 \quad \frac{\partial\left((1-\varepsilon) C_{s}\right)}{\partial t}=-D_{s} \nabla^{2}\left((1-\varepsilon) C_{s}\right)+a_{s} h_{m}\left(C_{a}-C_{s}\right)-(1-\varepsilon) r_{s}(6)$

148 The volume-averaged bound 1-MCP equation is:

$149 \frac{\partial C_{B}}{\partial t}=(1-\varepsilon) r_{s}$

$150 r_{s}$ is the rate of adsorption and was modelled by Ambaw et al. (2011)

$151 r_{s}=k_{a}\left(C_{\max }-C_{B}\right) C_{s}(8)$

152 Values of the model parameters are summarized in Table 1 and were obtained from previous

153 experiments (Ambaw et al., 2011).

154 The tortuosity $\tau$, in Eqn. 5, is defined as the ratio of linear distance between two points (say, point

1551 and point 2) both in the fluid phase to the actual length of diffusion path it takes to reach point 2

156 from point 1 . For randomly packed beds of spheres, the tortuosity can be estimated as a function

157 of porosity by (Liu and Masliyah, 2005):

$158 \tau=\left[\frac{(0.2+0.8 \varepsilon) \varepsilon^{20}}{\varepsilon^{13}+1.4 \times 10^{-8}(1-\varepsilon)}\right]^{1 / 16}$

159 The mass transfer coefficient between the fluid and solid phase $\left(h_{m}\right)$ consists of the resistance of

160 the apple skin mass transfer coefficient $\left(h_{s}\right)$ and the air film mass transfer coefficient $\left(h_{a}\right)$ and was

161 estimated using:

$162 \frac{1}{h_{m}}=\frac{1}{h_{s}}+\frac{1}{h_{a}}(10)$

163 The skin mass transfer coefficient is estimated from the effective diffusion coefficient of 1-MCP 164 in fruit tissue and the characteristic length, which in this case is the radius of the spherical fruit 
165 (Van der Sman, 2003). Taking the average radius to be $0.04 \mathrm{~m}$, the skin mass transfer coefficient

166 becomes $6.0 \times 10^{-7} \mathrm{~m} \mathrm{~s}^{-1}$. The air film mass transfer coefficient is estimated from correlation

167 equations. For Reynolds number ranging from 10 to 10000 for gases in a packed bed of sphere

168 recommended correlation equation with an average deviation of about $\pm 20 \%$ and a maximum of

$169 \pm 50 \%$ (Dwivedi \& Upadhyay, 1977) is

$170 \quad J_{D}=\frac{0.4548}{\varepsilon} \mathrm{Re}^{-0.4069}=\frac{h_{a}}{u}\left(\frac{\mu}{\rho D_{a}}\right)^{2 / 3}$

171 For the expected velocity range inside the stack, namely 0.001 to $0.1 \mathrm{~m} \mathrm{~s}^{-1}$, the air film mass

172 transfer coefficient ranges from $2.16 \times 10^{-4}$ to $3.32 \times 10^{-3} \mathrm{~m} \mathrm{~s}^{-1}$. Clearly, this is much larger than

173 the skin mass transfer coefficient. It can thus be expected that the uptake process by the fruit is

174 diffusion driven rather than convection driven.

175 The turbulent diffusion coefficient, through the empirical turbulent Schmidt number, is a function

176 of the turbulent viscosity $\left(D_{t}=\mu_{t} / \mathrm{Sc}_{\mathrm{t}}\right)$. In all CFD models of this study the SST k- $\omega$ turbulence

177 model was used for its superior performance over other steady Reynolds-averaged Navier-Stokes

178 turbulence models (Delele et al, 2008, Defraeye et al., 2012).

\section{$179 \quad$ 2.3.4 Geometry and computational domains}

180 Fig. 1a shows the geometry of the direct CFD model. The position, size and shape of the vent-

181 holes of the boxes are explicitly accounted. DE modeling was used to generate the geometry of

182 random stacking of 90 spheres (representing fruit) with diameter ranging from 80 to $85 \mathrm{~mm}$ in a

183 box (Tijskens et al., 2003). Fig. 1b shows the schematics of the porous medium model that is

184 equivalent to the direct CFD model. In this model, the details of the boxes and fruit were dropped

185 and replaced by the porous domain. 


\subsubsection{Boundary and initial conditions}

187 Fluid-solid interfaces were explicitly defined between the fluid and the solid domains for the

188 direct CFD model and set to a conservative interface flux for the scalar and no slip walls for 189 momentum transfer. In the porous medium model, fluid-porous interfaces were explicitly defined

190 between the fluid and porous domains and set to a conservative interface flux boundary for the

191 momentum and scalar conservation equations. In both models, internal walls of the container,

192 external surfaces of the circulation fan and external surface of the 1-MCP generator were set to

193 no slip walls. 1-MCP gas entering the domain was described by adding a boundary source at the

194 surface representing the outlet of the gas generator. The source is defined by a stepwise

195 decreasing 1-MCP flux term in such a way that $80 \%, 18 \%$ and $2 \%$ of the full 1-MCP dose enters

196 the domain within the first, second and third hours respectively, and zero during the remaining

197 period. The 1-MCP release profile from the generator was approximated from a separate

198 experiment by placing the generator in an empty container. The initial concentration of 1-MCP in

199 every domain in the model was equal to zero.

\section{$200 \quad$ 2.3.6 Simulation setup}

201 Simulations were performed using ANSYS-CFX-13 (ANSYS CFX, 2010), which uses the finite

202 volume method. The discretization and grid sensitivity studies of the direct CFD model were

203 discussed in our previous work (Ambaw et al. 2012). For the porous medium model, the grid

204 convergence index, which is based on Richardson extrapolation, was used (Journal of Fluids

205 Engineering, 2008). The optimum grid consisted of 23,000 cells for each box and a total of 206250,672 cells for the whole domain. 
207 For each problem, first, a steady state calculation was performed to obtain a converged solution

208 of the airflow. The steady state solutions were then used as initial values in the subsequent

209 transient scalar transport calculation.

\section{$210 \quad$ 2.3.7 Parameter sensitivity analysis}

211 The model parameters of the porous medium model are listed in Table 1. The purpose of the

212 sensitivity analysis was to quantify the relative importance of these parameters. The outputs of

213 interest in the present study are uniformity of the distribution of unbound 1-MCP and degree of

214 adsorption of the gas in the porous medium at intermediate time $(t=4 \mathrm{~h})$. At this intermediate

215 time there is some adsorption, which is anything but the saturation level, and it is within equitable

216 computing time. Uniformity of the gas distribution is measured as the standard deviation of the

217 local to the volume averaged unbound 1-MCP concentrations. Degree of adsorption is measured

218 as the ratio of volume averaged bound 1-MCP concentration during 4 hours to the maximum

219 adsorption capacity of apple fruits which is equal to $4.87 \times 10^{-6} \mathrm{~kg} \mathrm{~m}^{-3}$.

220 The sensitivity study was performed in two separate groups (Group 1 and Group 2, see Table 1).

221 Group 1 consist fluid flow parameters and Group 2 scalar transport parameters. The nominal

222 values and value ranges of the parameters included in the sensitivity analysis are summarized in

223 Table 1. In cases where the value range was not available or small, a range of $\pm 10 \%$ of the

224 nominal value was used.

225 The parameter sensitivity study was performed by using the design-of-experiment (DOE)

226 capability of the Design Exploration tool of ANSYS Workbench. The overall numbers of tests

227 (simulation runs) for the sensitivity analysis for each scenario were determined based on a 
228 Central Composite Design (CCD). A total of 45 and 85 simulation runs were instigated by the

229 CCD for Group 1 and Group 2, respectively.

$230 \quad 3$ Results and discussions

2313.1 Comparison of the porous medium and direct CFD model of the 232 storage container

\section{3.1.1 Airflow pattern}

234 Table 2 summarizes the linear and quadratic coefficients of the momentum loss model. The

235 pressure drop across the single box along the three directions, simulated using the direct CFD 236 models, are shown in Fig. $2 \mathrm{a}, \mathrm{b}$ and $\mathrm{c}$ for flow along $+\mathrm{x},+\mathrm{y}$ and $-\mathrm{z}$ directions respectively. The

237 resistances to flow (the pressure drop per unit length) across side faces (along the $\mathrm{x}$ - and $\mathrm{z}$-axis)

238 are equal, whereas, the resistance to vertical flow (along the y-axis) is different and considerably

239 higher. This is due to the small area fraction of vent holes at the bottom face of the box. The

240 direct CFD models helped to calculate and capture the anisotropic pressure losses effectively.

242 Fig. 3 shows velocity contour plots (color) and velocity direction (arrows) taken on a vertical

243 cutting plane passing through the centre of the stack. While the porous medium model predicts

244 averaged values, the direct CFD model provides more detailed information of airflow through

245 vent holes and in the air spaces between the fruits. The porous medium model reproduces the

246 highs and lows of the velocity field satisfactorily.

247 Fig. 4 shows the horizontal profiles of velocities obtained from direct CFD model (full curves)

248 and porous medium model (dashed curves) along with velocity measurement data (blue dots).

249 Higher velocities outside the stack and low velocities (mostly $<0.01 \mathrm{~m} / \mathrm{s}$ ) inside the stack are

250 well reproduced by the porous medium model. The special feature of the air flow in the region 
251 above fruits in the bottom box is, however, not properly captured by the porous medium model

252 (Fig. 4d). In this region, there is a characteristic channeled flow formed by the vent holes of the

253 box, and the restriction due to the bottom of the top box and the fruits from below. This is due to

254 the assumption of uniform porosity in the porous medium model.

\section{$255 \quad$ 3.1.2 1-MCP distribution}

256 Fig. 5a depicts simulated and measured values of 1-MCP concentration as free in air in the

257 container. Both the porous and direct CFD models predicted essentially uniform gas

258 concentration in the air. In confirmation, the measured values were also spatially uniform. The

259 observed spatial variability in the measurement was random with no characteristic trend and

260 within the measurement errors. There is, though, a slight off-set error with respect to the average

261 measured profile. The direct CFD model is, in average, 3\% and maximally 5\% over the average

262 measured values. The porous medium model is, in average, $4 \%$ and maximally $7 \%$ over the

263 average measured values. Difficulty in accurately capturing the geometry of stacked apple fruit in

264 a box, as well as several other model assumptions contributes to the error.

265 Fig. 5b depicts simulated unbound 1-MCP. Experimental determination of the magnitude of 266 unbound 1-MCP in fruit is almost impossible. The direct CFD model provides a framework to 267 examine the accuracy of the porous medium model in predicting the unbound in fruit

268 concentrations in time. Once more, the agreement between the two models is adequate with a

269 maximum difference of $10 \%$ during the early stage of the process. After about $10 \mathrm{~h}$ the two

270 models predicts similarly.

271 Concentration contours of 1-MCP that is free in air, unbound and bound in the fruit are shown in

272 Fig. 6. The plot depicts the level of detail information that the direct CFD model can provide as 
273 compared to the porous medium model. Both models predict a uniform 1-MCP distribution in the

274 air (Fig. 6 top row). The porous medium model predicts average distributions of unbound (Fig. 6

275 middle row) and bound (Fig.6 bottom row) 1-MCP concentrations across the porous domain

276 missing the distinct concentration gradients inside fruits. The direct CFD model calculates the

277 gradients of the 1-MCP concentration inside individual fruits.

278 The computation of the unbound and bound 1-MCP concentrations by the porous medium model

279 was to a great extent affected by the value of the mass transfer coefficient. Porous medium

280 simulations based on air film mass transfer coefficient only leads to erroneous prediction of the

281 bound 1-MCP concentration. Aspect of this observation is discussed below.

\section{$282 \quad 3.1 .3$ Effect of the mass transfer coefficient}

283 Comparisons of simulated volume-averaged bound 1-MCP concentrations of the porous medium

284 model based on air film mass transfer coefficient only (FMTC) (dash-dot curve) and air film and

285 skin mass transfer coefficients combined (F+SMTC) (dash-dot-dot curve) are shown in Fig. 7

286 together with the result obtained from the direct CFD model (full curve). This plot reveals that

287 FMTC overestimated the rate of adsorption (measured as the slop of the concentration vs. time 288 curve) significantly. The time to reach $90 \%$ saturation is estimated as 6,10 and 11 hours by the

289 FMTC, F+SMTC and direct CFD models respectively. The rationale in using the air film mass

290 transfer coefficient only was the assumption that the solid phase resistance would be captured by

291 the solid phase volume averaged transport equation. But, in the volume averaged equation, the

292 solid phase transport term becomes inconsequential to truly act as rate limiting. The high Biot

293 number (> 2) of the porous medium indicates the importance of solid phase resistance. The

294 incorporated skin mass transfer coefficient in essence corresponds to the solid phase resistance

295 and enables the porous medium model to better capture the adsorption profile. 


\subsection{Parameter sensitivity analysis}

297

298

299

300

301

302

303

\subsubsection{Sensitivity with respect to fluid flow parameters}

Fig. 8a and $b$ show the result of the sensitivity study of Group 1 parameters. The strong effect of porosity on degree of saturation and uniformity of gas distribution is evident. Porosity, in addition to describing the fluid flow, also defines the amount of adsorbent (solid volume) present in the medium. The inverse relationship with respect to degree of adsorption is in accordance to the way porosity related to the amount of adsorbent in the porous domain. Higher porosity means lower adsorbent volume (resulting in high 1-MCP-to-adsorbent ratio).

The turbulent Schmidt number, the viscous and inertial coefficients of the momentum loss models has minor effects on the degree of adsorption and uniformity of gas distribution. Particularly, the sensitivity with respect to coefficients of the momentum loss model along the yaxis is negligible. This suggests the possibility of simplifying the porous medium model by assuming an isotropic momentum loss model.

\subsubsection{Sensitivities with respect to the scalar transport parameters}

The relative importance of the scalar transport parameters on the degree of saturation (Fig. 8c), is sequenced as: $\mathrm{a}_{s}>h_{s}>k_{s}>\tau>D_{a}>h_{a}>D_{s}$. With respect to the uniformity of gas distribution (Fig. 7d), the parameters are sequenced as: $\mathrm{a}_{\mathrm{s}}>h_{s}>k_{s}>\tau>D_{s}>D_{a}>h_{a}$. The adsorption coefficient is inversely related to the uniformity of gas distribution. The specific area and the skin mass transfer coefficient have the stronger effects. These two parameters together establish the interfacial transfer; also, in essence $h_{s}$ define the solid phase diffusion process. This result confirms the rate determining role of the solid phase transport process. The diffusion of 1-MCP between fruits (taken into account by $D_{s}$ ) does not play a significant role in the uptake and distribution process. The rather minor influence of the tortuosity and air film mass transfer 
319 coefficient and the air diffusion coefficient are logical. These parameters characterize the fluid

320 phase transport process which is sufficiently fast in the process. Overall, the parameter sensitivity

321 analysis clearly shows the relative importance of the various processes in the gas transport.

\section{Conclusions}

323 A porous medium model for simulating the distribution of 1-MCP in boxes of fruit was

324 developed using the volume averaging technique. The reliability of the approach was assessed

325 through a thorough comparison of the porous medium model with an equivalent direct CFD

326 model and experimental measurements. Though the porous medium model cannot capture local

327 concentration gradients, it can be used to assess the condition of global gas distribution.

328 Through comprehensive parameter sensitivity analysis this work clearly showed where the

329 influential transport processes. Fluid flow parameters were observed to have negligible influence

330 on the simulation of the gas transport. Porosity, specific surface area, skin mass transfer

331 coefficient, and adsorption rate constant have strong influence on the porous medium simulation.

332 Based on this observation, one can consider model simplifications, for instance, by considering

333 isotropic instead of directional momentum loss models and also by excluding the diffusion

334 transport term from the volume averaged transport equation.

335 The results presented here are only valid for systems with similar dimensions to the $500 \mathrm{~L}$

336 container used in this study. When the dimensions are in a different order of magnitude, like in

337 commercial storage rooms for 200 to 500 tons of fruit, the relative time constants of the different

338 mechanisms may change and other mechanisms may become important. The study has; however,

339 shown the validity of the porous medium model, which can now be used to study such 
340 commercial systems. The approach developed is generic; it can be used to develop models of

341 transport of other gases in closed rooms with corresponding adjustment of model parameters.

\section{Acknowledgments}

343 The financial support of the Institute for the Promotion of Innovation by Science and Technology

344 in Flanders (project IWT 060720) is kindly appreciated. These projects received co-funding of

345 the Belgian Fruit and Vegetable Auctions. SmartFresh ${ }^{\mathrm{TM}}$ was kindly supplied for the

346 experimental trials by AgroFresh Inc. Prof. Opara's contributions were supported by the South

347 African Research Chairs Initiative of the Department of Science and Technology and National

348 Research Foundation, and the South African Postharvest Innovation Programme (PHI).

3496 References

350 Alvarez, G., \& Flick, D. (2007). Modelling turbulent flow and heat transfer using macro-porous 351 medium approach used to predict cooling kinetics of stack of food products. Journal of Food 352 Engineering, 80, 391-401

353 Ambaw, A., Verboven, P., Delele, M.A., Defraey, T., Tijskens, E., Schenk, A., Nicolai, B.N. 354 (2012a). CFD modeling of the 3D spatial and temporal distribution of 1-methylcyclopropene in

355 fruit storage container. Food and Bioprocess Technology, DOI: 10.1007/s11947-012-0913-7 356 Online First ${ }^{\mathrm{TM}}$

357 Ambawa, A., Delele, M.A., Defraeye T., Ho, Q.T., Opara, L.U., Nicolai, B.M., Verboven, P. 358 (2012 b). The use of CFD to characterize and design post-harvest storage facilities: Past, present 359 and future. Comput. Electron. Agric., http://dx.doi.org/10.1016/j.compag. 2012. 05.009.

360 Ambaw, A., Beaudry, R., Bulens, I., Delele, M.A., Ho, Q.T., Schenk, A., Nicolai, B.N., 361 Verboven, P. (2011). Modeling the diffusion-adsorption kinetics of 1-methylcyclopropene (1$362 \mathrm{MCP})$ in apple fruit and non- target materials in storage rooms. Journal of Food Engineering, 363 102, 257-265.

364 ANSYS CFX-Solver Theory Guide (Release 12.1). ANSYS Inc, Southpointe, 275 Technology 365 Drive, Canonsburg, PA 15317.

366 Blankenship, S.M., Dole, J.M. (2003). 1-Methylcyclopropene: a review. Postharvest Biology and 367 Technology, 28, 1-25. 
Defraeye, T., Verboven, P., Nicolai, B.M. (2012). CFD modelling of flow and scalar exchange of spherical food products: Turbulence and boundary-layer modelling. Journal of Food 370 Engineering, 114, 495-504

371 Delele, M.A., Schenk, A., Tijskens, E., Ramon, H., Nicolai, B.M., Verboven, P. (2009).

372 Optimization of the humidification of cold stores by pressurized water atomizers based on a multiscale CFD model. Journal of Food Engineering, 91, 228-239

374 Delele, M.A., Tijskens, E., Atalay, Y.T., Ho, Q.T., Ramon, H., Nicolai, B.M., Verboven, P. 375 (2008). Combined Discrete Element and CFD Modelling of airflow through random stacking of 376 horticultural products in vented boxes. Journal of Food Engineering, 89 (1), 33 - 41

377 Delele, M.A., Verboven, P., Ho, Q.T., Nicolai, B.M. (2010). Advances in mathematical 378 modelling of postharvest refrigeration processes. Stewart Postharvest Review. 2:1

379 Dwivedi, P.N., and Upadhyay, S.N. (1977). Ind. Eng. Chem., Proc. Des. Dev., 16, 157

380 Hassanizadeh, M., Gray, W.G. (1979). General conservation equations for multi-phase systems: 381 1. Averaging procedure. Advance in Water Resources, vol. 2, 131-41.

382 Hoang, M., Verboven, P., Baelmans, M., Nicolai, B.M. (2003). A continuum model for airflow, 383 heat and mass transfer in bulk of chicory roots. Transactions of the ASAE, 46, 1603-1611.

384 J. Fluids Eng. (2008). Procedure for Estimation and Reporting of Uncertainty Due to 385 Discretization in CFD Applications. July 2008, Volume 130, Issue 7, 078001 (4 pages). 386 http://dx.doi.org/1.2960953.

387 Lerew, L.E. (1978). Development of a temperature-weight loss model for bulk stored potatoes, 388 PhD thesis, Michigan State University, East Lansing, MI, 1978.

393 Nahor, H.B., Hoang, M.L., Verboven, P., Baelmans, M., Nicolai, B.M. (2005). CFD model of the 394 airflow, heat and mass transfer in cool stores, International Journal of Refrigeration, 28, 368-380

395 Quintard, M., Whitaker, S. (2005). Coupled, Nonlinear Mass Transfer and Heterogeneous 396 Reaction in Porous medium, in: Kambiz Vafai, Handbook of porous medium $\left(2^{\text {nd }}\right.$ Eds.). Taylor \& 397 Francis Group, LLC, pp. 3-39.

398 Sisler, E.C., Blankenship, S.M. (1996). Methods of counteracting an ethylene response in plants. 399 United States Patent, No 5518988 (in English).

400 Tijskens, E., Ramon, H., De Baerdemaeker, J. (2003). Discrete element modelling for process 401 simulation in agriculture. Journal of Sound and Vibration, 266, 493-514. 
402 Tominaga, Y., Stathopoulos, T. (2007). Turbulent Schmidt numbers for CFD analysis with

403 various types of flow field. Atmospheric Environment, 41, 8091-8099.

404 Vallejo, F., Beaudry, R. (2006). Depletion of 1-MCP by 'non-target' materials from fruit storage 405 facilities. Postharvest Biology and Technology, 40, 177-182.

406 Van der Sman, R.G.M. (2003). Simple model for estimating heat and mass transfer in regular407 shaped foods. Journal of Food Engineering, 60: 383-390.

408 Verboven, P., Flick, D., Nicolai, B.M., Alvarez, G. (2006). Modeling transport phenomena in 409 refrigerated food bulks, packages and stacks: basics and advances. International journal of 410 refrigeration, 29 (6), 985-997.

411 Whitaker, S. (1999). The Method of Volume Averaging. Dordrecht: Kluwer Academic Press.

412 Zou, Q., Opara, LU., McKibbin, R. (2006a). A CFD modeling system for airflow and heat 413 transfer in ventilated packaging for fresh foods: I: Initial analysis and development of 414 mathematical models. Journal of Food Engineering. 77, 1037-1047.

415 Zou, Q., Opara, LU., McKibbin R. (2006b). A CFD modeling system for airflow and heat 416 transfer in ventilated packaging for fresh foods: II: Computational solution, software 417 development, and model testing. Journal of Food Engineering. 77, 048-1058.

\section{Nomenclature}

\section{Roman symbols}

$a_{\text {s }} \quad$ Specific surface area of bulk apple fruits, $6(1-\varepsilon) / d \quad \mathrm{~m}^{2} / \mathrm{m}^{3}$

$C_{a} \quad$ Concentration of 1-MCP in air $\quad \mathrm{kg} / \mathrm{m}^{3}$

$C_{B} \quad$ Concentration of bound 1-MCP in fruit $\quad \mathrm{kg} / \mathrm{m}^{3}$

$C_{\max } \quad$ 1-MCP adsorption capacity of apple fruit $\quad \mathrm{kg} / \mathrm{m}^{3}$

$C_{R 1 i} \quad$ Linear Resistance Coefficient $\quad \mathrm{kg} \mathrm{m}^{-3} \mathrm{~s}^{-1}$

$C_{R 2 i} \quad$ Quadratic Resistance Coefficient $\quad \mathrm{kg} \mathrm{m}^{-4}$

$C_{s} \quad$ Concentration of bound 1-MCP in wood $\mathrm{kg} / \mathrm{m}^{3}$

d Average diameter of apple fruits m

$D_{a} \quad$ Diffusion coefficient of 1-MCP in air $\quad \mathrm{m}^{2} / \mathrm{s}$

$D_{s} \quad$ Effective diffusion coefficient of 1-MCP inside fruits $\quad \mathrm{m}^{2} / \mathrm{s}$

$D_{t} \quad$ Turbulent Diffusion coefficient $\quad \mathrm{m}^{2} / \mathrm{s}$

$d V \quad$ Volume of the representative elemental volume 


$\begin{array}{lll}d V_{a} & \text { Volume occupied by air phase in the elemental volume } & \\ h_{a} & \text { Air film mass transfer coefficient } & \mathrm{m} / \mathrm{s} \\ h_{m} & \text { Overall mass transfer coefficient in the stack } & \mathrm{m} / \mathrm{s} \\ h_{s} & \text { Skin mass transfer coefficient } & \mathrm{m} / \mathrm{s} \\ J_{D} & \text { Dimensionless factor } & \\ k & \text { Turbulence kinetic energy } & \mathrm{m}^{2} / \mathrm{s}^{2} \\ p & \text { Pressure } & \mathrm{Pa} \\ \operatorname{Re} & \text { Reynold number } & \\ r_{s} & \text { Rate of 1-MCP adsorption in apple fruit } & \mathrm{kg} \mathrm{m} \mathrm{s}^{-1} \\ S_{u} & \text { Momentum source term } & \mathrm{kg} \mathrm{m} \mathrm{s}^{-2} \\ t & \text { Time } & \mathrm{s} \\ \mathbf{U} & \text { Vector of velocity, } \mathbf{V}_{\mathrm{x}, \mathrm{y}, \mathrm{z}} & \mathrm{m} / \mathrm{s}^{-2} \\ u & \text { Velocity magnitude } & \mathrm{m} / \mathrm{s}^{2}\end{array}$

\section{Greek symbols}

$\varepsilon \quad$ Porosity of stacked apple fruits given

$\mu \quad$ Dynamic viscosity

$\mathrm{kg} \mathrm{m}^{-1} \mathrm{~s}^{-1}$

$\mu_{t} \quad$ Turbulent eddy viscosity

$\mathrm{kg} \mathrm{m}^{-1} \mathrm{~s}^{-1}$

$\mu_{\text {eff }} \quad$ Effective viscosity $\left(=\mu+\mu_{t}\right)$

$\mathrm{kg} \mathrm{m}^{-1} \mathrm{~s}^{-1}$

$\rho_{a} \quad$ Density of dry air

$\mathrm{Kg} \mathrm{m}^{-3}$

$\delta \quad$ Identity matrix or Kronecker Delta function

$\tau \quad$ Tortuosity

\section{Sub and super-scripts}

$\begin{array}{ll}a & \text { Air } \\ \max & \text { Maximum } \\ s & \text { Solid } \\ t & \text { Turbulent } \\ \mathbf{T} & \text { The transform operator }\end{array}$




\section{Tables}

423 Table 1 Nominal values and value ranges of the model parameters used for the sensitivity study

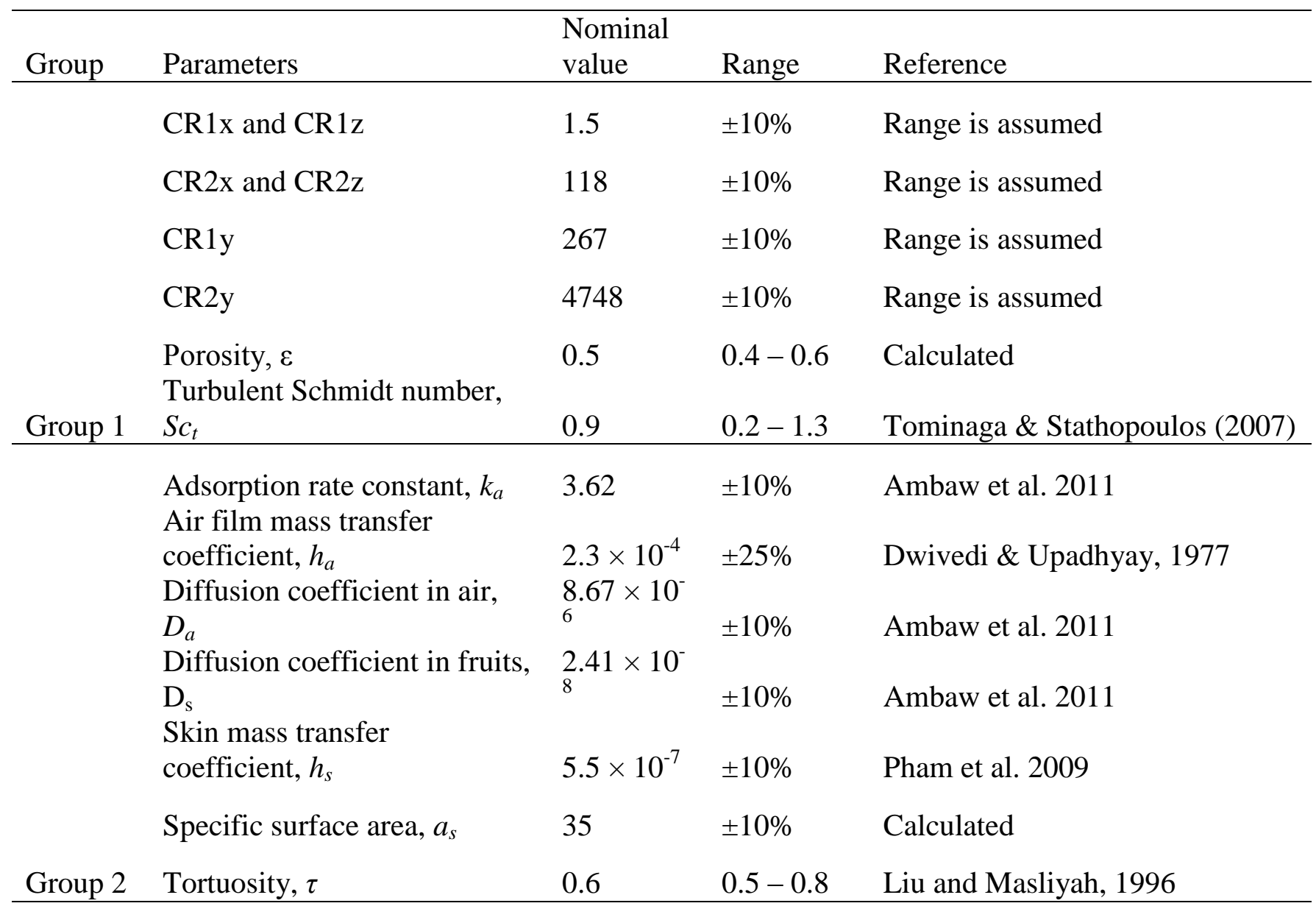

425 Table 2 Linear and quadratic coefficients of the airflow resistance model of the porous medium, 426 obtained from direct CFD simulation

\begin{tabular}{lcc}
\hline Direction & $C_{R 1}$ & $C_{R 2}$ \\
\hline $\mathrm{x}$ & 1.5 & 118 \\
$\mathrm{y}$ & 262 & 4748 \\
$\mathrm{z}$ & 1.5 & 118 \\
\hline
\end{tabular}


(a)

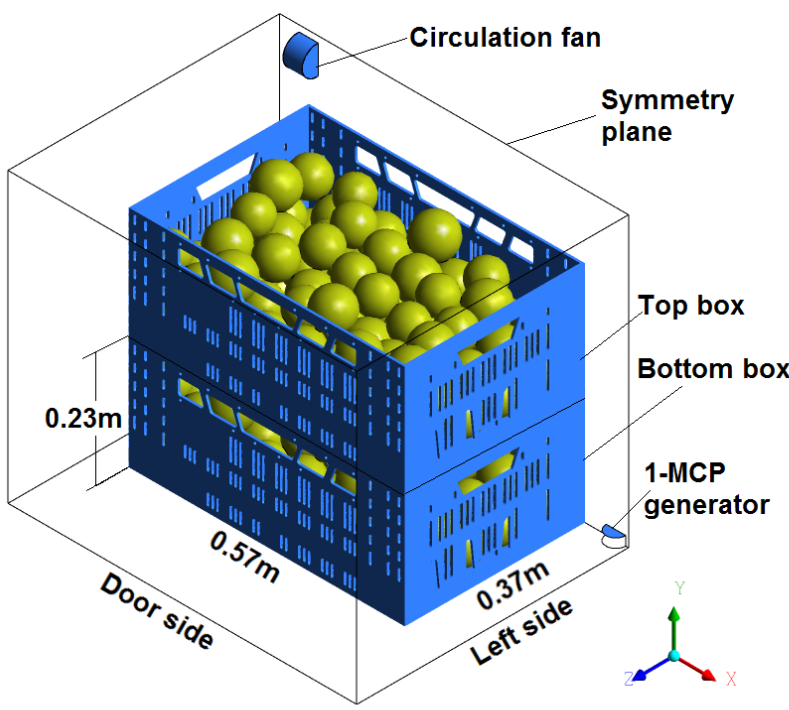

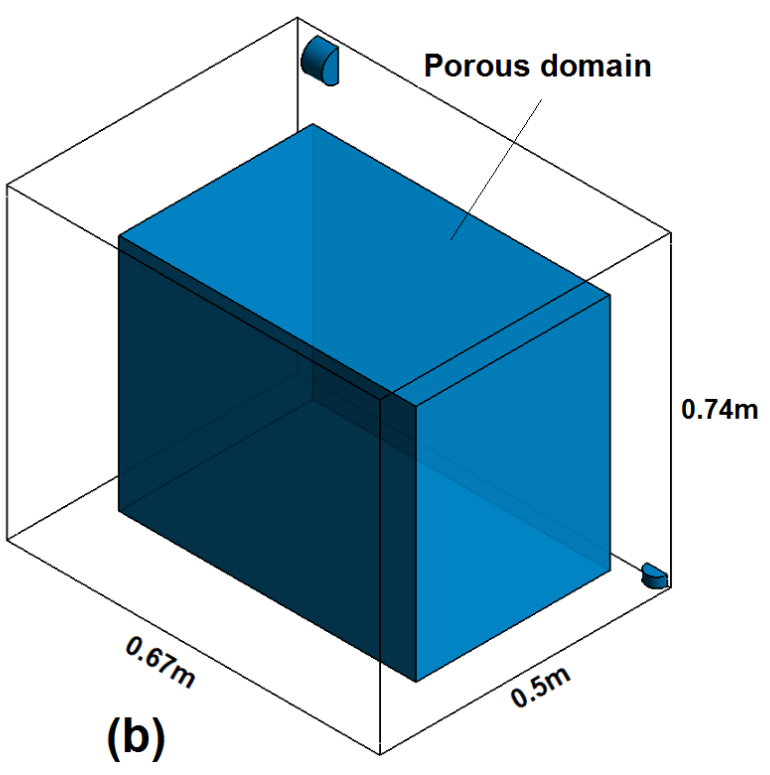

(b)
Fig. 1. Schematics of the model geometry for studying 1-MCP treatment in a $500 \mathrm{~L}$ container: direct CFD model (a) and porous medium model (b). The model geometry considers only half of the full experimental setup by assuming symmetry.

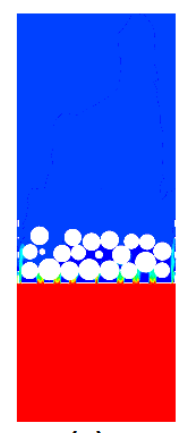

(b)

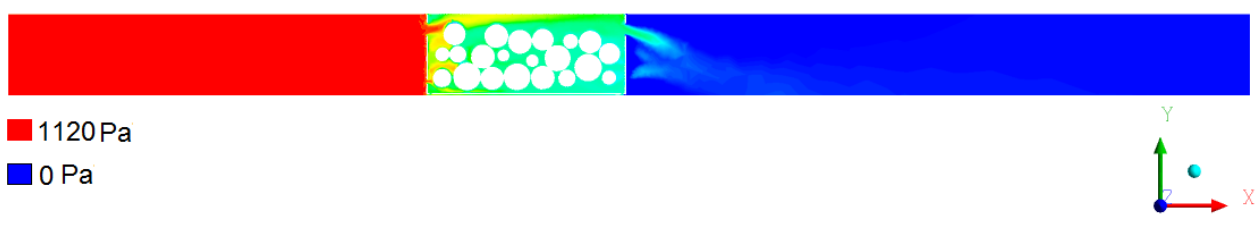

(c)

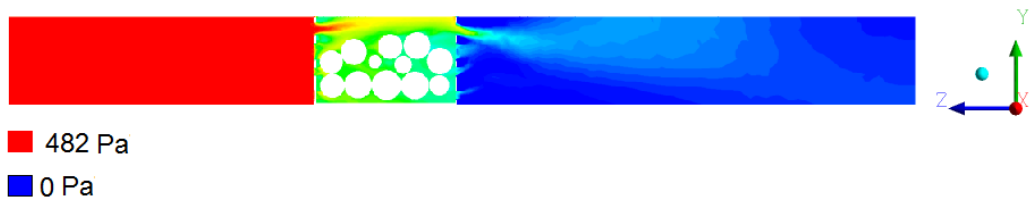

(a)

Fig. 2. Simulated pressured drops across single box using the direct CFD model. Flow from bottom to top of the box (along y axis) (a), flow perpendicular to the smaller side face of the box (along $\mathrm{x}$-axis) (b) and flow perpendicular to the larger side face of the box (along $-\mathrm{z}$ ) (c). The contour is shown on a plane bisecting the stack. Superficial velocity for these particular contours was $4 \mathrm{~m} \mathrm{~s}^{-1}$. 

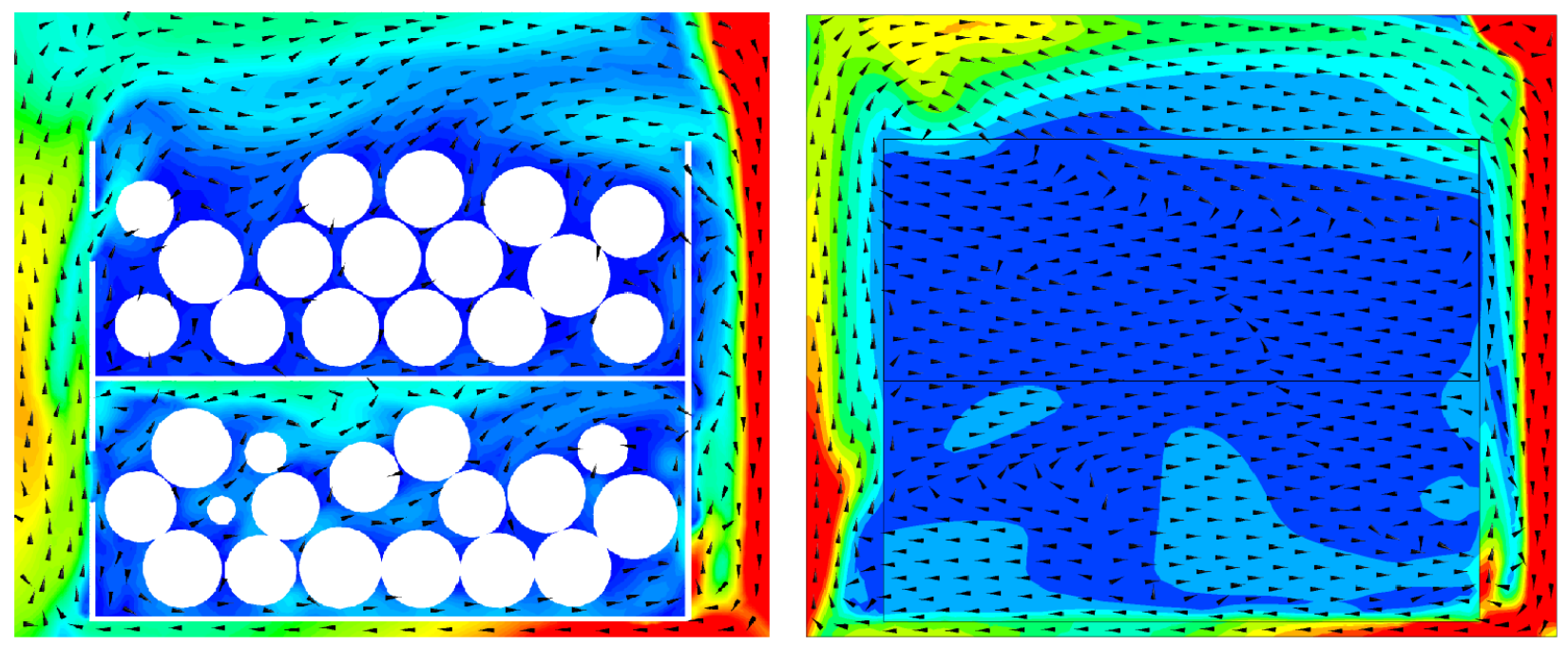

447 Fig. 3. Velocity contour and airflow direction on vertical plane (parallel to the symmetry plane) 448 through the center of the boxes in the storage container: direct CFD model (left) and porous 449 medium model (right). 

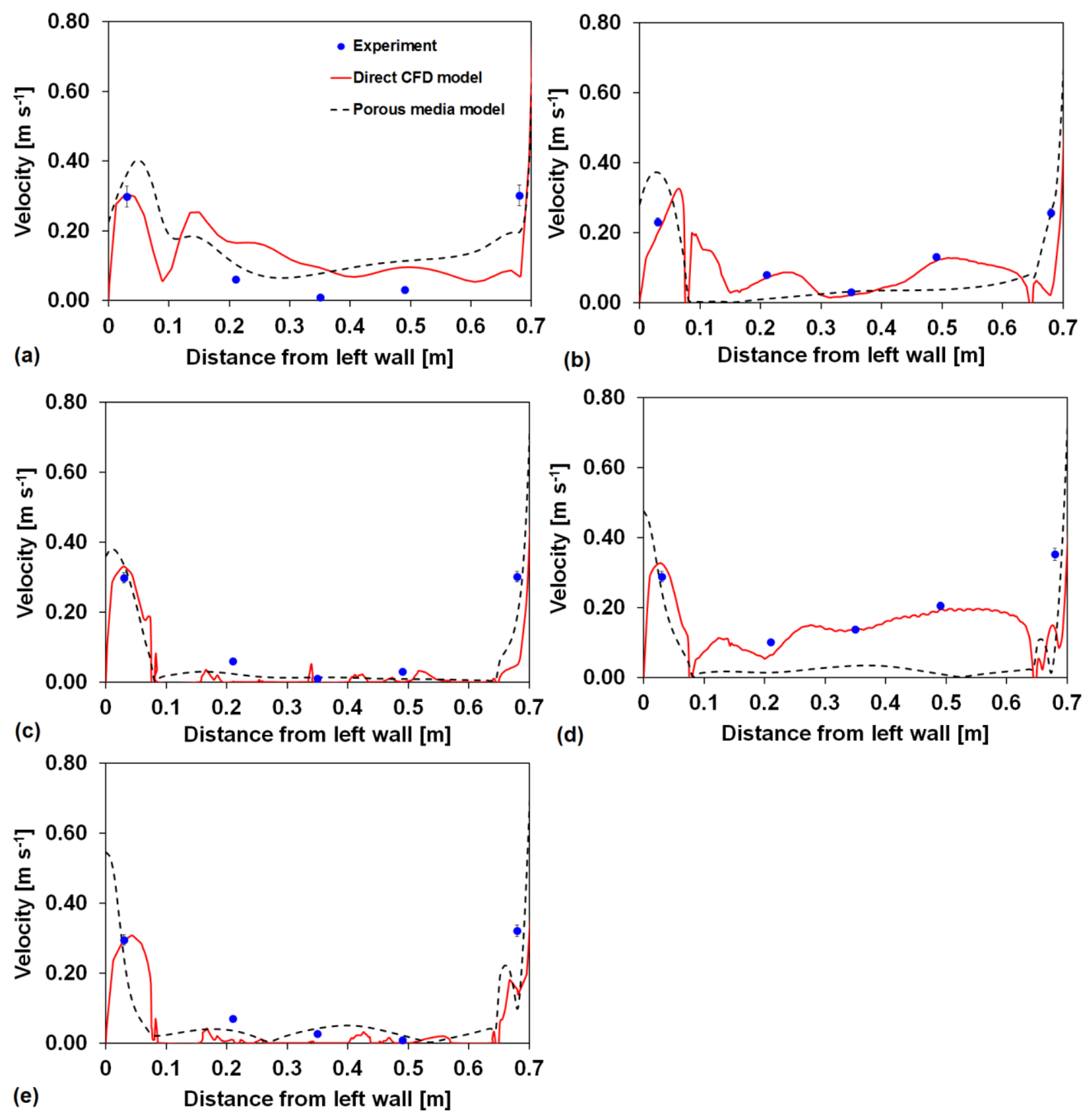

452 Fig. 4. Measured (blue dots) and calculated by the direct CFD model (full curve) and porous 453 medium model (broken curve) velocity magnitudes across the region above the stack (a), upper 455 region inside the top box (b), middle region inside the top box (c), upper region inside the bottom 456 box $(d)$ and middle region inside the bottom box (e). 

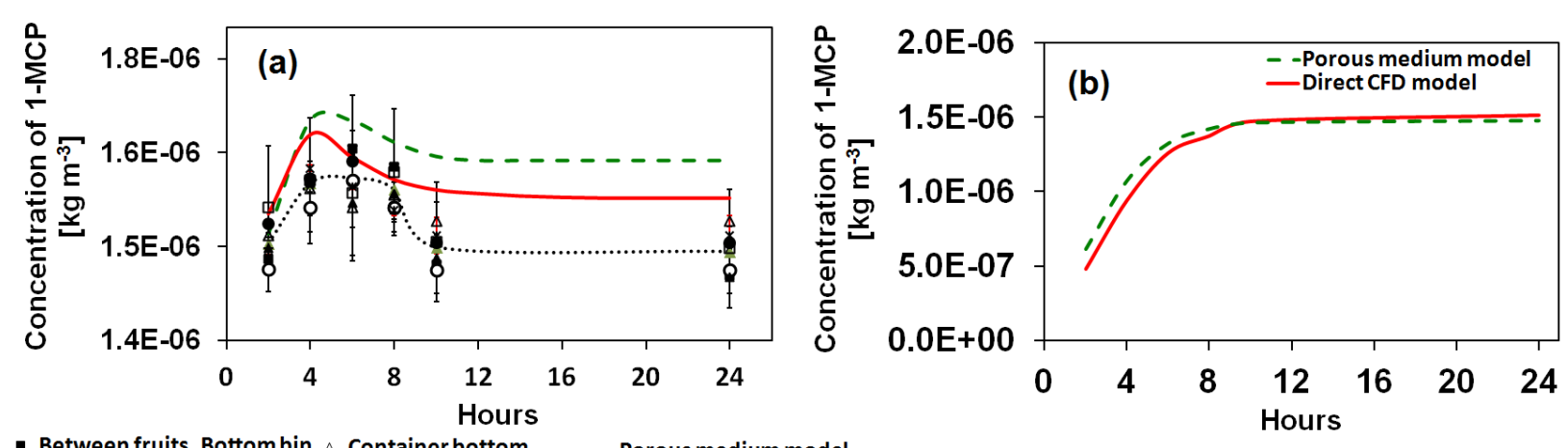

458 Fig. 5. Simulation of profiles of concentration of 1-MCP in the air (a), unbound 1-MCP in the 459 fruit (b) using direct CFD model (full curve) and porous medium model (dashed curves). The 460 curves are for values averaged over the boxes. Symbols in (a) are measurement values of 461 462 463

- Between fruits_Bottom bin $\triangle$ Container bottom - -Porous medium model

+ Left side

○ Symmetry

$\square$ Right side

- Container top

- Direct CFD model

$\triangle$ Front side

..... Measurement average

* Between fruits_Top bin samples. The 1-MCP dose was $1 \mu \mathrm{L} / \mathrm{L}$, and the temperature was $1^{\circ} \mathrm{C}$.

464

465

466

467

468

469
Porous medium model

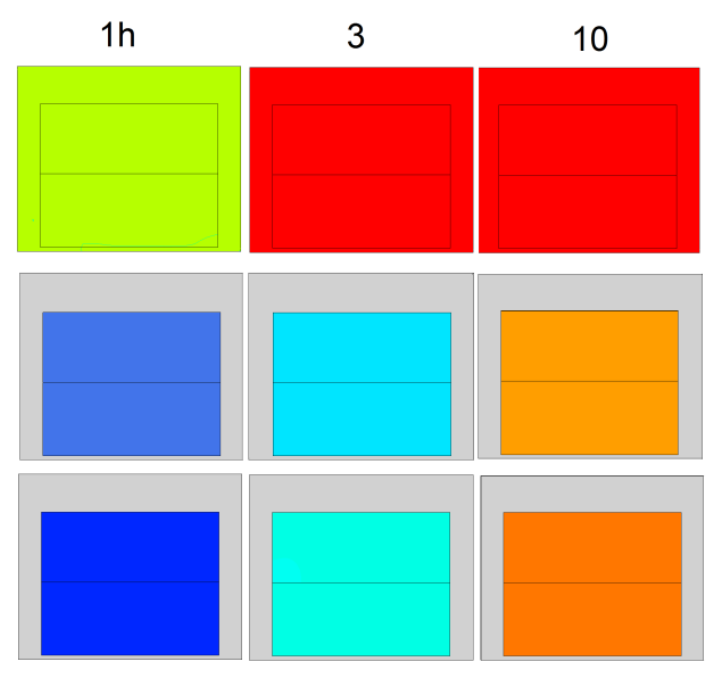

Direct CFD model

1h
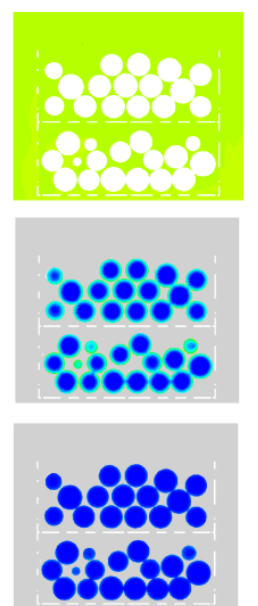

3
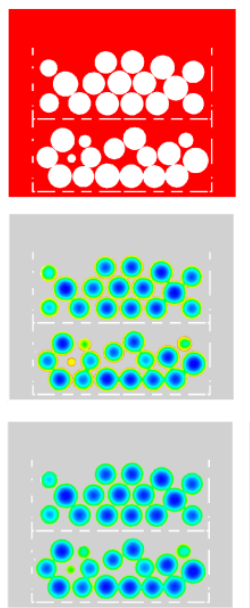

10
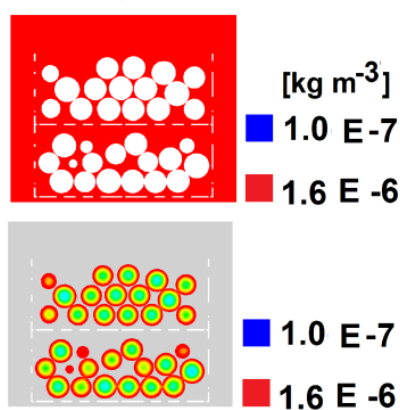

$1.0 \mathrm{E}-7$

$1.6 \mathrm{E}-6$

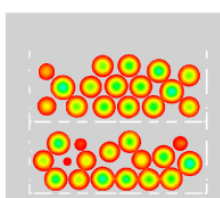

$1.0 \mathrm{E}-7$

4.8 E - 6

Fig. 6. Contour of free in air (top row), unbound in fruits (middle raw) and bound in fruits (bottom raw) on a vertical section through center of the boxes from the porous medium and direct CFD models at three different times. 


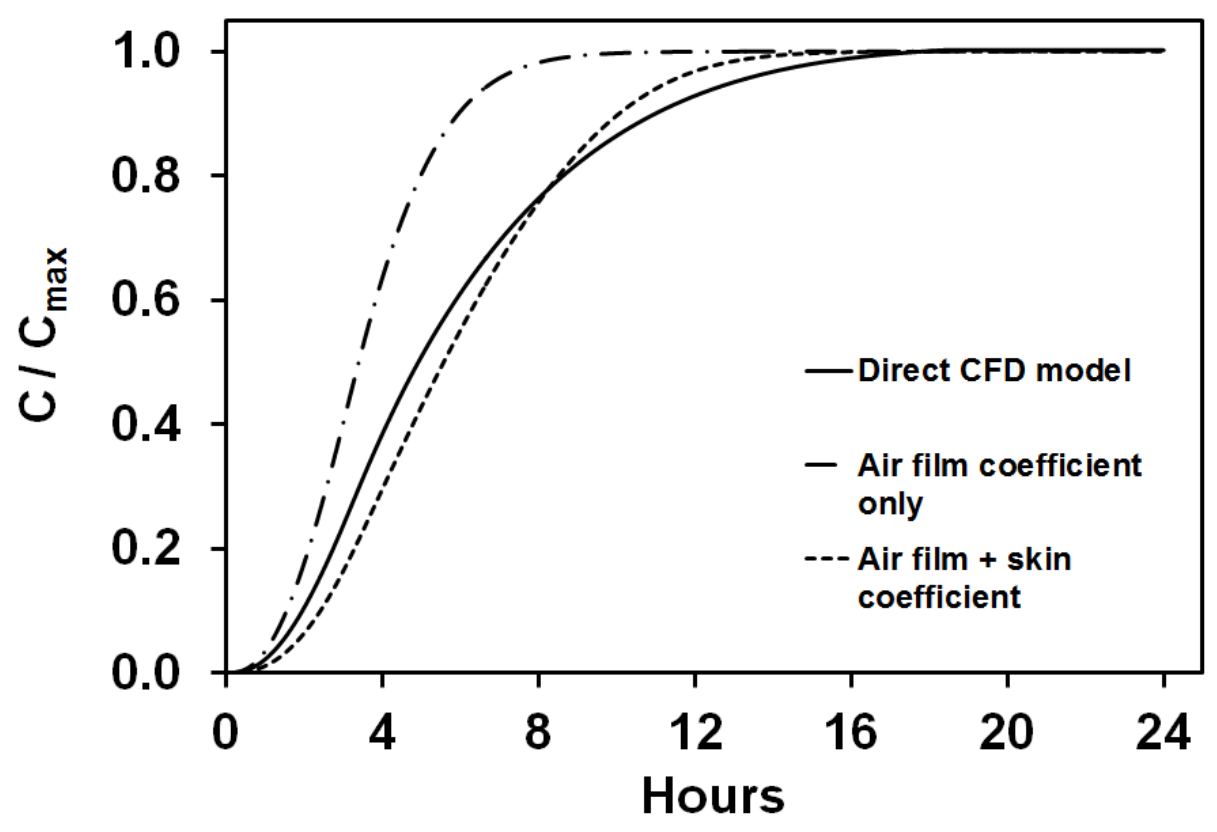

471 Fig. 7. Comparison of the time dependent bound 1-MCP concentration (averaged over the boxes) 472 calculated by the porous medium model with air film mass transfer coefficient only (dash-dot 473 curve), air film and skin mass transfer coefficient combined (dash curve) and by the direct CFD 474 model (full curve). 

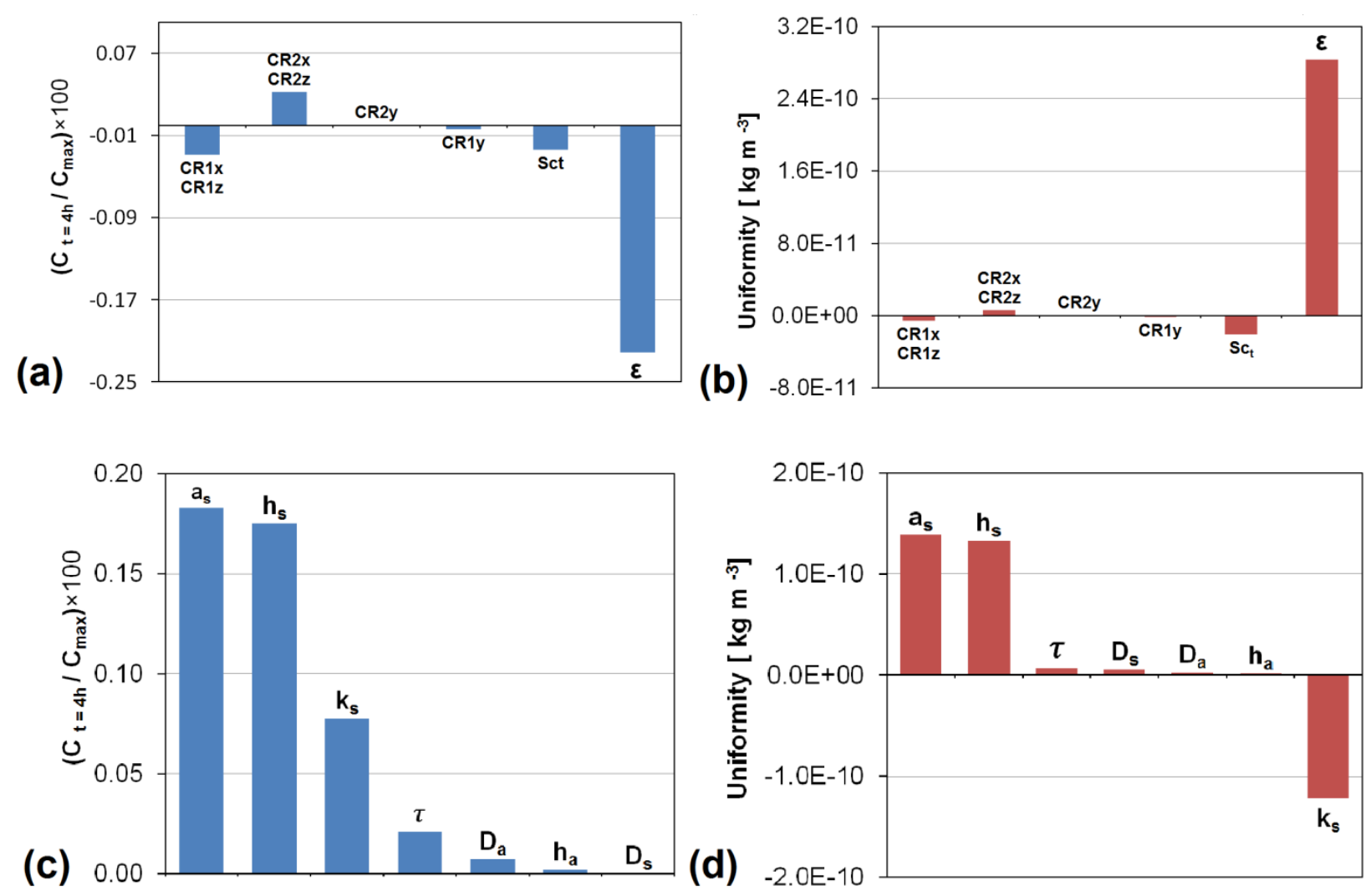

477 Fig. 8. Bar graphs showing sensitivities of the porous medium model simulations to Group 1 478 parameters ( top row) and Group 2 parameters (bottom row) in predicting degree of saturation 479 (left column) and uniformity of gas distribution (right column).

480 